\title{
Capacitated Vehicle Routing Problem Solving using Adaptive Sweep and Velocity Tentative PSO
}

\author{
M. A. H. Akhand, Zahrul Jannat Peya \\ Dept. of Computer Science and Engineering Khulna \\ University of Engineering \& Technology \\ Khulna 9203, Bangladesh
}

\author{
Kazuyuki Murase \\ Graduate School of Engineering \\ University of Fukui \\ Fukui 910-8507, Japan
}

\begin{abstract}
Vehicle Routing Problem (VRP) has become an integral part in logistic operations which determines optimal routes for several vehicles to serve customers. The basic version of VRP is Capacitated VRP (CVRP) which considers equal capacities for all vehicles. The objective of CVRP is to minimize the total traveling distance of all vehicles to serve all the customers. Various methods are used to solve CVRP, among them the most popular way is splitting the task into two different phases: assigning customers under different vehicles and then finding optimal route of each vehicle. Sweep clustering algorithm is well studied for clustering nodes. On the other hand, route optimization is simply a traveling salesman problem (TSP) and a number of TSP optimization methods are applied for this purpose. In Sweep, cluster formation staring angle is identified as an element of CVRP performance. In this study, a heuristic approach is developed to identify appropriate starting angle in Sweep clustering. The proposed heuristic approach considers angle difference of consecutive nodes and distance between the nodes as well as distances from the depot. On the other hand, velocity tentative particle swarm optimization (VTPSO), the most recent TSP method, is considered for route optimization. Finally, proposed adaptive Sweep (i.e., Sweep with proposed heuristic) plus VTPSO is tested on a large number of benchmark CVRP problems and is revealed as an effective CVRP solving method while outcomes compared with other prominent methods.
\end{abstract}

Keywords-Capacitated vehicle routing problem; Sweep clustering and velocity tentative particle swarm optimization

\section{INTRODUCTION}

Vehicle Routing Problem (VRP) has become an integral part in logistic operations which determines optimal routes for several vehicles to serve customers [1]. A proper selection of vehicle routes is very important to promote the economic benefits in operations. VRP is a hard optimization task to minimize total traveling distance of all the vehicles to serve all the customers from a depot. The general constrains of VRP are each customer is serviced exactly once (by a single vehicle) and total load of a route does not exceed capacity of the assigned vehicle [2].

The basic version of VRP is Capacitated VRP (CVRP) which considers equal capacities for all the vehicles [3], [4], [6]. The simplest form of CVRP considers one depot and vehicles depart from the depot at the beginning and return to the depot at the end. In CVRP, all customers have known demands and known locations for the delivery.
CVRP is a complex optimization task and its objective is to minimize the total traveling distance for all vehicles to serve all customers. Mathematically, a CVRP is defined as

$$
\begin{aligned}
& \text { Minimize } \sum_{i \in N} \sum_{j \in N} \sum_{v \in V} C_{i j} X_{i j}^{v} \\
& \text { Subject to } \sum_{v \in V} y_{i}^{v}=1 \text { for } i \in N \\
& \sum_{i \in N} x_{i j}^{v}=y_{j}^{v} \text { for } j \in N \text { and } v \in V \\
& \sum_{j \in N} x_{i j}^{v}=y_{i}^{v} \text { for } i \in N \text { and } v \in V \\
& \sum_{i \in N} d_{i} y_{i}^{v} \leq Q \text { for } v \in V \\
& \sum_{i \in N} x_{i 1}^{v} \leq 1 \text { for } v \in V \\
& \sum_{j \in N} x_{1 j}^{v} \leq 1 \text { for } v \in V
\end{aligned}
$$

In this formulation, the objective function is expressed by (1) which states that the total traveling distance of all vehicles (i.e., CVRP cost) is to be minimized. Equation (2) represents the constraint that each customer must be visited once by one vehicle, where $y_{i}^{v}=1$ if vehicle $v$ visits customer $i$ otherwise it is zero. It is guaranteed in (3) and (4) that each customer is visited and left with the same vehicle, where $x_{i j}^{v}=1$ if vehicle $v$ travels from customer $i$ to customer $j$, and 0 otherwise. A constraint in (5) ensures that the total delivery demands of vehicle $v$ do not exceed the vehicle capacity. Equations (6) and (7) express that vehicle availability should not be exceeded.

Various methods have been investigated to solve CVRP in last few decades. A number of methods are available that optimizes customer assignment under vehicles and routes of the vehicles together [5]. On the other hand, the most popular way of solving CVRP is splitting the task into two different phases: firstly, assigning customers under different vehicles and secondly, finding optimal route for each vehicle [2]. 
Among several ways for customer node assignment, Sweep clustering algorithm is well studied due to its simplicity. The algorithm calculates polar angles of all the nodes and then assigns nodes into different clusters according to their angles [5], [10]. The algorithm can be implemented using two different methods, forward Sweep (i.e., anti-clockwise) and backward Sweep (i.e., clock wise) [8]. On the other hand, route optimization is simply a traveling salesman problem (TSP) and a TSP optimization method is employed for this purpose, in general [8], [9].

A number of CVRP studies are available using traditional TSP optimization methods with Sweep clustering. Nurcahyo et al. [8] investigated a Sweep based VRP for public transport of Semarang, Indonesia. Both forward Sweep and backward Sweep are considered for clustering; and route generation is accomplished through nearest neighbour algorithm of TSP. Han and Tabata [9] used Genetic Algorithm (GA) with Sweep algorithm to solve CVRP. In the method, a chromosome of GA is considered as a complete CVRP solution that is prepared from Sweep outcome. Suthikarnnarunai [7] used integer programming to generate TSP routes of Sweep clusters. Author also induced 2-opt exchange to improve a VRP solution exchanging nodes between tours. Aziz et al. [13] is also investigated nearest neighbour algorithm with Sweep clustering to solve CVRP.

Recently, a number of nature inspired swarm intelligence methods are investigated to generate vehicle route as the methods are found efficient to solve TSP. Yousefikhoshbakht and Khorram [11] used ant colony optimization (ACO) on Sweep clusters and then 3-opt local search are used for improving the VRP solutions. Reed et al. [12] investigated ACO with k-means clustering to solve the CVRP associated with collection of recycling waste from households. Venkatesan et al. [14] investigated Particle Swarm Optimization (PSO) to generate vehicle tour from Sweep clusters. PSO is also investigated in CVRP by Pornsing [4].

The objective of this study is to investigate effective CVRP solving method through adaptive Sweep where cluster starting angle is adaptive to problem. The most of the Sweep based methods, including the already discussed methods, considered standard Sweep for assigning customers under different vehicles and employed different methods to generate optimal routes for the vehicles. In standard Sweep, cluster formation starts from $0^{\circ}$ and consequently advances toward $360^{\circ}$ to assign all the nodes under different vehicles [7]. Problem with such rigid starting is identified that total clusters formation may exceeds total number of available vehicles for some instances. And, starting from different user-defined angles identified better clustering and hence achieved better CVRP solution [17]. In this study, a heuristic approach is developed to identify appropriate starting angle in Sweep clustering. On the other hand, velocity tentative particle swarm optimization (VTPSO), the most recent TSP method, is considered for route optimization. Finally, proposed adaptive Sweep plus VTPSO is tested on a large number of benchmark CVRP problems and outcomes are compared with other prominent methods.
The outline of the remaining paper is as follows. Section II explains the proposed CVRP solving method with adaptive Sweep and VTPSO. Section III is for experimental studies which presents outcomes of the proposed method in solving benchmark CVRPs as well as compares with other related methods. At last, Section IV gives a brief conclusion of the paper.

\section{SOLVING CVRP USING ADAPTIVE SWEEP AND VELOCITY TENTATIVE PSO (VTPSO)}

This section explains proposed CVRP solving method using adaptive Sweep and VTPSO. At first it explains proposed adaptive Sweep clustering. To make the paper selfcontained, VTPSO, the considered TSP route optimization method, is also explained briefly.

\section{A. Clutering using Adaptive Sweep}

Appropriate starting angle for cluster formation is an important matter in Sweep algorithm. Existing studies checked different fixed starting angles. But such trial and check method is required to set for every individual problem [17]. Therefore, as an alternative, a heuristic method is investigated in this study which aim is to identify the appropriate cluster formation starting angle $(\theta s)$ for a given problem.

The proposed heuristic approach considers angle difference of consecutive nodes in angle basis ordered node list $(O N L)$; and distance between the nodes and distances from the depot. The approach first calculates preference value $(p \theta)$ of each consecutive nodes and maximum $p \Theta$ is considered as the outcome of starting angle $(\theta s)$. Suppose the depot and other two consecutive nodes are D, N1 and N2, respectively. Polar angles of the nodes are $\theta 1$ and $\theta 2$. The distances of the nodes from the depot are $d N 1$ and $d N 2$; and distance between the nodes is $d N 12$. Fig. 1 shows the graphical representation of the matter for better understanding. Preference value $(p \theta)$ for the starting angle between the nodes $\mathrm{N} 1$ and $\mathrm{N} 2$ means to place the nodes in two different clusters and is calculated using (8).

$$
p \Theta=\alpha^{*}(\theta 2-\theta 1)+\beta *\{d N 12+\operatorname{Min}(d N 1, d N 2)\}
$$

In the equation, $\alpha$ and $\beta$ are the arbitrary constants to emphasis angle difference and node distances, respectively. According to first part of (8), the preference value increases with angular difference of the nodes (i.e., $\theta 2-\theta 1$ ). The second part of the equation is minimum distance to travel the two nodes from depot. The outcome of the equation (i.e., $p \Theta$ value) will be large if both the nodes are far from the depot as well as distance between them is large. On the other hand, $p \Theta$ value will be low even larger angle difference when both the nodes are closed to depot. After calculating the $p \Theta$ values for all the consecutive nodes, the maximum value is considered as the starting angle. If $p \Theta$ value for nodes $N 1$ and $N 2$ is found maximum then cluster formation will be start from $N 2$ for anti-clock wise cluster formation. Motivation of such starting is that these two nodes might not be same cluster. Staring from $\mathrm{N} 2$, cluster formation consequently advances assigning nodes into clusters considering vehicle capacity like standard Sweep. In such case N1 will be assigned in the last cluster. 


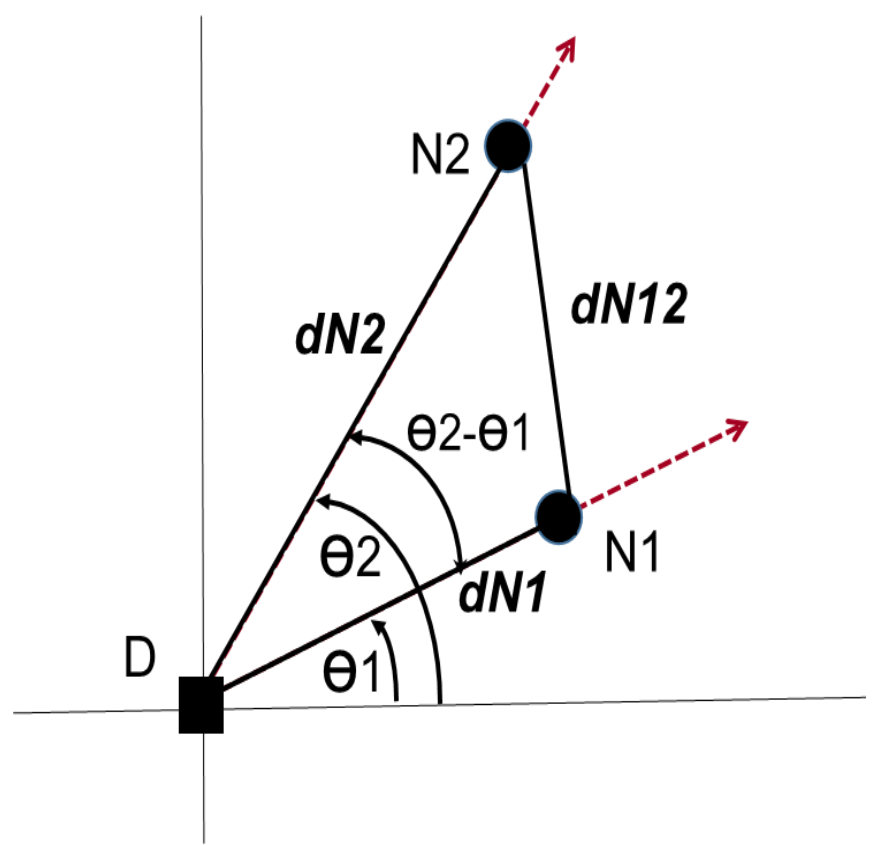

Fig. 1. Demonstration of start angle formation in adaptive Sweep.

\section{B. Route Generation using VTPSO}

VTPSO [15] is the recent swarm intelligence method to solve TSP extending Particle Swarm Optimization (PSO). In PSO, every particle represents a tour and changes its tour at every iteration with velocity calculated considering the best tour encountered before by itself (called as particle best) and the best tour encountered by the swarm (called as global best). Swap Sequence (SS) and Swap operator (SO) based operation is considered for velocity calculation. A SO indicates two cities in a tour those positions will be swapped. Suppose, a TSP problem has ten cities and a solution is 1-2-3-6-4-5-7-89-10. A $S O(4,6)$ gives the new solution S'.

$$
\begin{aligned}
S^{\prime}= & S+S O(4,6) \\
= & (1-2-3-6-4-5-7-8-9-10)+S O(4,6) \\
& =1-2-3-5-4-6-7-8-9-10
\end{aligned}
$$

Here ' + ' means to apply $\mathrm{SO}(\mathrm{s})$ on the solution.

A swap sequence is made up of one or more swap operators.

$$
S S=\left(\mathrm{SO}_{1}, \mathrm{SO}_{2}, \mathrm{SO}_{3}, \ldots S \mathrm{SO}_{n}\right),
$$

Where, $\mathrm{SO}_{1}, \mathrm{SO}_{2}, \mathrm{SO}_{3}, \ldots \mathrm{SO}_{n}$ are the swap operators. Implementation of a SS means apply all the SOs on the solution in order. In traditional PSO, the new tour of TSP is considered after applying all the SOs of a SS and no intermediate measure is considered. On the other hand, VTPSO considers the calculated velocity SS as a tentative velocity and conceives a measure called partial search (PS) to apply calculated SS to update particle's position (i.e., TSP tour).

VTPSO calculates velocity SS as like other PSO based methods. At each iteration step, it calculates velocity SS using
(11) considering i) last applied velocity $\left(v^{(t-l)}\right)$, ii) previous best solution of the particle $\left(P_{i}\right)$, and iii) global best solution of the swarm $(G)$.

$$
V_{i}^{(t)}=V_{i}^{(t-1)} \otimes \alpha\left(P_{i}-X_{i}^{(t-1)}\right) \otimes \beta\left(G-X_{i}^{(t-1)}\right) \alpha, \beta \epsilon[1,0]
$$

Through PS technique, VTPSO measures performance of tours applying SOs of the calculated SS one after another, and the final velocity is considered for which it gives better tour. Therefore, PS technique explores the option of getting better tour considering the intermediate tours with a SS applying its SOs one by one.

$$
\begin{aligned}
& \quad \text { Suppose } V_{i}^{(t)}=S O_{1}, S O_{2}, S O_{3}, \ldots S O_{n} \text { then in PS } \\
& X_{i}^{1(t)}=X_{i}^{(t-1)}+S O_{1} \\
& X_{i}^{2(t)}=X_{i}^{1(t)}+S O_{2}=X_{i}^{(t-1)}+S O_{1}+S O_{2} \\
& X_{i}^{n(t)}=X_{i}^{n-1(t)}+S O_{n}
\end{aligned}
$$

In the above cases $X_{i}^{1(t)}, X_{i}^{2(t)}, \ldots \ldots, X_{i}^{n(t)}$ are the tentative intermediate tours; and the final tour $X_{i}^{(t)}$ in PS is the tentative tour having the minimum tour cost.

$$
X_{i}^{(t)}=X_{i}^{j(t)},
$$

Where, $X_{i}^{j(t)}$ provides the minimum tour cost among $X_{i}^{1(t)}, X_{i}^{2(t)}, \ldots X_{i}^{j(t)} \ldots X_{i}^{n(t)}$. Finally, the velocity considered as $V_{i}^{(t)}=S O_{1}, S O_{2}, S O_{3}, \ldots S O_{j} 1<\mathrm{j} \leq \mathrm{n}$. The detailed description of VTPSO for TSP is available in [15].

\section{EXPERIMENTAL STUDIES}

This section experimentally investigates the efficacy of proposed adaptive Sweep algorithm to cluster customers and VTPSO for route generation on a set of benchmark CVRP problems. A detailed observation has also given on a selected problem for better understanding of the way of performance improvement in proposed method.

\section{A. Bench Mark Data and General Experimental Methodology}

In this study, total 51 benchmark CVRPs have been considered from two different sets of Augerat benchmark problems which are A-VRP and P-VRP [16]. In A-VRP, number of customer (i.e., nodes) varies from 32 to 80 , total demand varies from 407 to 932 , number of vehicle varies from 5 to 10 and capacity of individual is 100 for all the problems. For example, A-n $32-\mathrm{k} 5$ has 32 customers and 5 vehicles. On the other hand, in P-VRP, number of customer varies from 16 to 101 , total demand varies from 246 to 22500 and vehicle capacity varies from 35 to 3000 . Tables I and II show the brief description of the A-VRP and P-VRP benchmark problems, respectively. Two numeric values in a problem name present the number of nodes and vehicles associated with the problem. The detailed description of the problems is available in provider's website ${ }^{1}$. According to Tables I and II, the selected benchmark problems belong to large varieties in number of nodes, vehicles and demands; and therefore, provide a diverse test bed.

\footnotetext{
${ }^{1}$ http://neo.lcc.uma.es/vrp/vrp-instances/
} 
TABLE I. DESCRIPTION OF A-VRP BENCHMARK PROBLEMS FOR CVRP

\begin{tabular}{|c|c|c|c|c|c|}
\hline SI & $\begin{array}{l}\text { Problem } \\
\text { Name }\end{array}$ & $\begin{array}{l}\text { Total } \\
\text { Nodes }\end{array}$ & \begin{tabular}{|l} 
Numb \\
er of \\
Vehicl \\
e
\end{tabular} & $\begin{array}{l}\text { Individual Vehicle } \\
\text { Capacity }\end{array}$ & $\begin{array}{l}\text { Total } \\
\text { Demand }\end{array}$ \\
\hline \begin{tabular}{|l|}
1 \\
\end{tabular} & A-n32-k5 & 32 & 5 & 100 & 410 \\
\hline 2 & A-n33-k5 & 33 & 5 & 100 & 446 \\
\hline 3 & A-n33-k6 & 33 & 6 & 100 & 541 \\
\hline \begin{tabular}{|l|}
4 \\
\end{tabular} & A-n34-k5 & 34 & 5 & 100 & 460 \\
\hline 5 & A-n36-k5 & 36 & 5 & 100 & 442 \\
\hline \begin{tabular}{|l|}
6 \\
\end{tabular} & A-n37-k5 & 37 & 5 & 100 & 407 \\
\hline \begin{tabular}{|l|}
7 \\
\end{tabular} & A-n37-k6 & 37 & 6 & 100 & 570 \\
\hline 8 & A-n38-k5 & 38 & 5 & 100 & 481 \\
\hline \begin{tabular}{|l|}
9 \\
\end{tabular} & A-n39-k5 & 39 & 5 & 100 & 475 \\
\hline 1 & A-n39-k6 & 39 & 6 & 100 & 526 \\
\hline 1 & A-n44-k6 & 44 & 6 & 100 & 570 \\
\hline \begin{tabular}{|l|}
1 \\
\end{tabular} & A-n45-k6 & 45 & 6 & 100 & 593 \\
\hline 1 & A-n45-k7 & 45 & 7 & 100 & 634 \\
\hline \begin{tabular}{|l|}
1 \\
\end{tabular} & A-n46-k7 & 46 & 7 & 100 & 603 \\
\hline 1 & A-n48-k7 & 48 & 7 & 100 & 626 \\
\hline \begin{tabular}{|l|}
1 \\
\end{tabular} & A-n53-k7 & 53 & 7 & 100 & 664 \\
\hline \begin{tabular}{|l|}
1 \\
\end{tabular} & A-n54-k7 & 54 & 7 & 100 & 669 \\
\hline \begin{tabular}{|l|}
1 \\
\end{tabular} & A-n55-k9 & 55 & 9 & 100 & 839 \\
\hline 1 & A-n60-k9 & 60 & 9 & 100 & 829 \\
\hline 2 & A-n61-k9 & 61 & 9 & 100 & 885 \\
\hline 2 & A-n62-k8 & 62 & 8 & 100 & 733 \\
\hline \begin{tabular}{|l|}
2 \\
\end{tabular} & A-n63-k9 & 63 & 9 & 100 & 873 \\
\hline 2 & A-n63-k10 & 63 & 10 & 100 & 932 \\
\hline \begin{tabular}{|l|}
2 \\
\end{tabular} & A-n64-k9 & 64 & 9 & 100 & 848 \\
\hline 2 & A-n65-k9 & 65 & 9 & 100 & 877 \\
\hline \begin{tabular}{|l|}
2 \\
\end{tabular} & A-n69-k9 & 69 & 9 & 100 & 845 \\
\hline \begin{tabular}{|l|}
2 \\
\end{tabular} & A-n80-k10 & 80 & 10 & 100 & 942 \\
\hline
\end{tabular}

TABLE II. DESCRIPTION OF P-VRP BENCHMARK PROBLEMS FOR CVRP

\begin{tabular}{|l|l|l|l|l|l|}
\hline $\begin{array}{l}\text { SI } \\
\cdot\end{array}$ & $\begin{array}{l}\text { Problem } \\
\text { Name }\end{array}$ & $\begin{array}{l}\text { Total } \\
\text { Nodes }\end{array}$ & $\begin{array}{l}\text { Numb } \\
\text { er of }\end{array}$ & $\begin{array}{l}\text { Individual Vehicle } \\
\text { Capacity }\end{array}$ & $\begin{array}{l}\text { Total } \\
\text { Demand }\end{array}$ \\
\hline 1 & P-n16-k8 & 16 & 8 & 35 & 246 \\
\hline 2 & P-n19-k2 & 19 & 2 & 160 & 310 \\
\hline 3 & P-n20-k2 & 20 & 2 & 160 & 310 \\
\hline 4 & P-n21-k2 & 21 & 2 & 160 & 298 \\
\hline 5 & P-n22-k2 & 22 & 2 & 160 & 308 \\
\hline 6 & P-n22-k8 & 22 & 8 & 3000 & 22500 \\
\hline 7 & P-n23-k8 & 23 & 8 & 40 & 313 \\
\hline 8 & P-n40-k5 & 40 & 5 & 140 & 618 \\
\hline 9 & P-n45-k5 & 45 & 5 & 150 & 692 \\
\hline 1 & P-n50-k7 & 50 & 7 & 150 & 951 \\
\hline 1 & P-n50-k8 & 50 & 8 & 120 & 951 \\
\hline 1 & P-n50-k10 & 50 & 10 & 100 & 951 \\
\hline 1 & P-n51-k10 & 51 & 10 & 80 & 777 \\
\hline 1 & P-n55-k7 & 55 & 7 & 170 & 1042 \\
\hline 1 & P-n55-k8 & 55 & 8 & 160 & 1042 \\
\hline 1 & P-n55-k10 & 55 & 10 & 115 & 1042 \\
\hline 1 & P-n55-k15 & 55 & 15 & 70 & 1042 \\
\hline 1 & P-n60-k10 & 60 & 10 & 120 & 1134 \\
\hline 1 & P-n60-k15 & 60 & 15 & 80 & 1134 \\
\hline 2 & P-n65-k10 & 65 & 10 & 130 & 1219 \\
\hline 2 & P-n70-k10 & 70 & 10 & 135 & 1313 \\
\hline 2 & P-n76-k4 & 76 & 4 & 350 & 1364 \\
\hline 2 & P-n76-k5 & 76 & 5 & 280 & 1458 \\
\hline 2 & P-n101-k4 & 101 & 4 & 400 & \\
\hline
\end{tabular}

Benchmark problems are required to preprocess to use in the experiments. A customer is represented as a co-ordinate in a problem. Coordinates are updated considering depot as $[0,0]$ for easy calculation. Distance matrix is prepared using the coordinates. Polar angle of each customer is calculated for angle based sweep operation. Standard Sweep (i.e., $\theta_{s}=0^{0}$ ) does not have any parameter to set and it starts cluster formation from $0^{0}$ (i.e., $\theta_{s}=0^{\circ}$ ). In adaptive Sweep, the values of $\alpha$ and $\beta$ were set to 0.6 and 0.2 , respectively and found effective for most of the problems. In few other problems $\alpha$ and $\beta$ values are tuned between 0.2 and 0.6. Both anti clock and clock wise sweep operations are considered in both standard and adaptive Sweep algorithm. The experiments have been done on a PC (Intel Core i5-3470 CPU @ 3.20 GHz CPU, 4GB RAM) with Windows 7 OS.

\section{B. Detailed Experimental Observation on a Selected Problem}

This section presents detailed results for A-n53-k7 problem. In route optimization with VTPSO, the population size and number of iteration were set 100 and 200, respectively. For better understanding, experiments conducted for standard Sweep $\left(\theta_{s=} 0^{0}\right)$ along with adaptively selected angle.

Fig. 2 is the graphical representation of the solutions of An53-k7 problem for standard Sweep plus VTPSO and adaptive Sweep plus VTPSO. In standard Sweep (Fig. 2(a)) nodes are divided into eight clusters and Cluster 8 is for remaining three nodes having total demand 29 only although vehicle capacity is 100. On the other hand, total CVRP demand are fulfilled by seven clusters by adaptive Sweep through adaptively selected $\theta_{s}=220.6^{0}$ (Fig. 2(b)) to start from node 3. It is visible from the figure that angle difference between nodes 33 and 3 is large and both are relatively far from depot. It is observed from the figure that several clusters are common in both solutions. Clusters 3, 4, 5, 6 and 7 of Fig. 2(a) are similar to clusters 7, 1, 2, 3 and 4 of Fig. 2(b), respectively. On the other hand, nodes of clusters 1, 2 and 8 of Fig. 2(a) are optimally assigned into clusters 5 and 6 in Fig. 2(b). With same VTPSO route optimization and summing up the individual tour costs, CVRP cost for standard Sweep and adaptive Sweep are 1174 and 1090, respectively. The figure clearly revealed the effectiveness of adaptive Sweep on CVRP outcome since both the cases VTPSO is used for individual vehicle route generation.

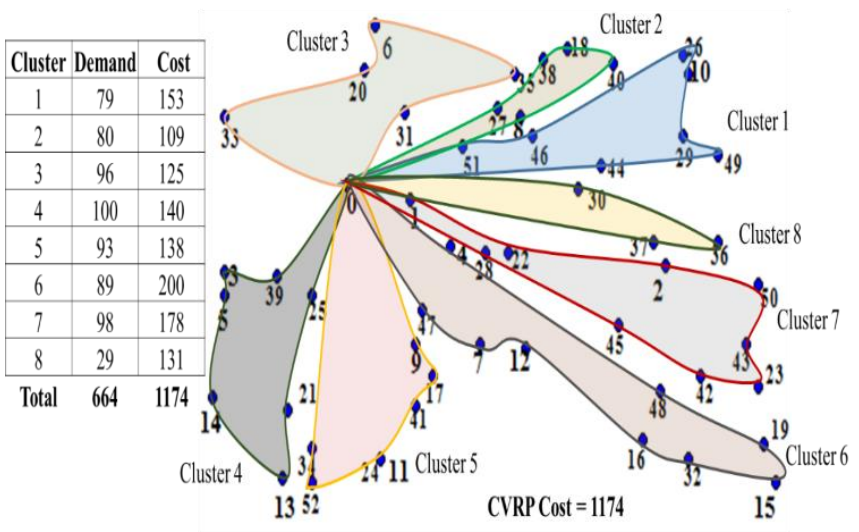

(a) Solution for standard Sweep (i.e., $\theta s=0^{\circ}$ ) and VTPSO. 


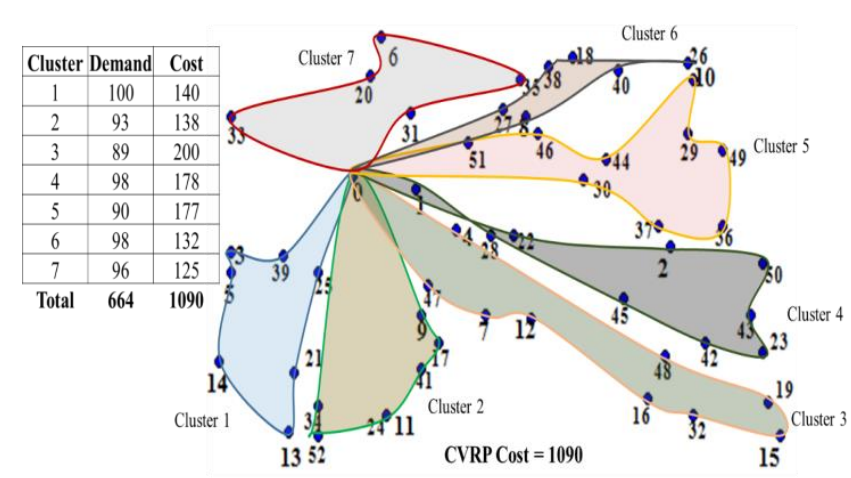

(b) Solution for adaptive Sweep with $\theta s=220.6^{\circ}$ and VTPSO.

Fig. 2. Graphical representation of A-n53-k7 solution with standard Sweep and adaptive Sweep clustering with VTPSO.

\section{Experimental Results and Performance Comparison}

This section first identifies the proficiency of adaptive Sweep clustering over standard Sweep clustering in solving benchmark CVRPs. For the fair comparison, the population size and the number of iteration of VTPSO were 100 and 200, respectively. The selected parameters are not optimal values, but considered for simplicity as well as for fairness in observation. Finally, outcomes of the proposed method compared with the prominent methods.

Tables III and IV compare CVRP costs for clustering with standard Sweep and adaptive Sweep on A-VRP and P-VRP benchmark problems, respectively. Bottom of the tables shows average and Win/Draw/Lose summary. In adaptive Sweep, cluster formation starting angle is problem dependent and selected through proposed heuristic approach. Therefore, the starting angle is different for different problems as seen in the tables. On the other hand, standard Sweep is for only Sweep clustering with $\theta_{s}=0^{0}$.

TABLE III. CVRP COST COMPARISON FOR CLUSTERING WITH STANDARD SWEEP AND ADAPTIVE SWEEP ON A-VRP BENCHMARK PROBLEMS

\begin{tabular}{|l|l||l||l|l|}
\hline \multirow{2}{*}{ Sl. } & \multirow{2}{*}{ Problem } & $\begin{array}{l}\text { Standard } \\
\text { Sweep }\left(\Theta_{s}=\mathbf{0}^{\mathbf{0}}\right) \\
\text { + VTPSO }\end{array}$ & \multicolumn{2}{|l|}{ Adaptive Sweep + VTPSO } \\
\cline { 3 - 5 } & & CVRP Cost & $\begin{array}{l}\text { Starting Angle } \\
\left(\Theta_{s}\right)\end{array}$ & CVRP Cost \\
\hline 1 & A-n32-k5 & 882 & 152.02 & 882 \\
\hline 2 & A-n33-k5 & 788 & 195.95 & 698 \\
\hline 3 & A-n33-k6 & 874 & 303.18 & 751 \\
\hline 4 & A-n34-k5 & 867 & 203.2 & 785 \\
\hline 5 & A-n36-k5 & 942 & 323.13 & 881 \\
\hline 6 & A-n37-k5 & 795 & 248.84 & 754 \\
\hline 7 & A-n37-k6 & 1131 & 264.29 & 1112 \\
\hline 8 & A-n38-k5 & 857 & 148.57 & 813 \\
\hline 9 & A-n39-k5 & 877 & 180 & 877 \\
\hline 10 & A-n39-k6 & 991 & 246.8 & 972 \\
\hline 11 & A-n44-k6 & 1164 & 253.3 & 1056 \\
\hline 12 & A-n45-k6 & 1115 & 138.01 & 1073 \\
\hline 13 & A-n45-k7 & 1305 & 180 & 1305 \\
\hline 14 & A-n46-k7 & 983 & 75.96 & 975 \\
\hline 15 & A-n48-k7 & 1152 & 3.18 & 1152 \\
\hline 16 & A-n53-k7 & 1174 & 220.6 & 1090 \\
\hline
\end{tabular}

\begin{tabular}{|l|l||l||l|l|}
\hline 17 & A-n54-k7 & 1361 & 4.09 & 1361 \\
\hline 18 & A-n55-k9 & 1190 & 318.96 & 1190 \\
\hline 19 & A-n60-k9 & 1552 & 170.54 & 1503 \\
\hline 20 & A-n61-k9 & 1219 & 333.43 & 1164 \\
\hline 21 & A-n62-k8 & 1532 & 263.66 & 1408 \\
\hline 22 & A-n63-k9 & 1823 & 153.43 & 1823 \\
\hline 23 & A-n63-k10 & 1477 & 6.34 & 1477 \\
\hline 24 & A-n64-k9 & 1598 & 94.57 & 1598 \\
\hline 25 & A-n65-k9 & 1368 & 237.99 & 1317 \\
\hline 26 & A-n69-k9 & 1254 & 352.09 & 1259 \\
\hline 27 & A-n80-k10 & 2136 & 149.04 & 2136 \\
\hline \hline Average & $\mathbf{1 2 0 0 . 2 6}$ & & $\mathbf{1 1 6 3 . 4 1}$ \\
\hline $\begin{array}{l}\text { Win/Draw/Lose Summary of adaptive Sweep over } \\
\text { standard Sweep }\end{array}$ & $\mathbf{1 6 / 1 0 / 1}$ \\
\hline
\end{tabular}

TABLE IV. CVRP COST COMPARISON FOR CLUSTERING WITH STANDARD SWEEP AND ADAPTIVE SWEEP ON P-VRP BENCHMARK PROBLEMS

\begin{tabular}{|c|c|c|c|c|}
\hline \multirow{2}{*}{ Sl. } & \multirow{2}{*}{ Problem } & \multirow{2}{*}{\begin{tabular}{|c}
$\begin{array}{c}\text { Standard } \\
\text { Sweep }\left(\theta_{s}=0^{0}\right) \\
+ \text { VTPSO }\end{array}$ \\
CVRP Cost
\end{tabular}} & \multicolumn{2}{|c|}{ Adaptive Sweep + VTPSO } \\
\hline & & & $\begin{array}{c}\text { Starting Angle } \\
\left(\Theta_{s}\right)\end{array}$ & CVRP Cost \\
\hline 1 & P-n16-k8 & 545 & 335.1 & 549 \\
\hline 2 & P-n19-k2 & 236 & 335.1 & 246 \\
\hline 3 & P-n20-k2 & 238 & 335.1 & 249 \\
\hline 4 & P-n21-k2 & 238 & 335.1 & 211 \\
\hline 5 & P-n22-k2 & 237 & 335.1 & 216 \\
\hline 6 & P-n22-k8 & 668 & 238.39 & 633 \\
\hline 7 & P-n23-k8 & 687 & 333.43 & 634 \\
\hline 8 & P-n40-k5 & 492 & 119.48 & 483 \\
\hline 9 & P-n45-k5 & 528 & 119.48 & 524 \\
\hline 10 & P-n50-k7 & 585 & 278.43 & 583 \\
\hline 11 & P-n50-k8 & 690 & 278.43 & 677 \\
\hline 12 & P-n50-k10 & 783 & 278.43 & 783 \\
\hline 13 & P-n51-k10 & 804 & 208.3 & 802 \\
\hline 14 & P-n55-k7 & 602 & 278.43 & 595 \\
\hline 15 & P-n55-k8 & 609 & 242.59 & 586 \\
\hline 16 & P-n55-k10 & 742 & 278.43 & 745 \\
\hline 17 & P-n55-k15 & 1133 & 278.43 & 1099 \\
\hline 18 & P-n60-k10 & 835 & 278.43 & 830 \\
\hline 19 & P-n60-k15 & 1092 & 278.43 & 1119 \\
\hline 20 & P-n65-k10 & 864 & 278.43 & 859 \\
\hline 21 & P-n70-k10 & 900 & 278.43 & 911 \\
\hline 22 & P-n76-k4 & 605 & 104.04 & 612 \\
\hline 23 & P-n76-k5 & 655 & 144.16 & 647 \\
\hline 24 & P-n101-k4 & 721 & 115.46 & 699 \\
\hline \multicolumn{2}{|c|}{ Average } & 645.38 & & 637.17 \\
\hline \multicolumn{4}{|c|}{$\begin{array}{l}\text { Win/Draw/Lose Summary of adaptive Sweep over } \\
\text { standard Sweep }\end{array}$} & $16 / 1 / 7$ \\
\hline
\end{tabular}


TABLE V. CVRP COST COMPARISON WITH EXISTING METHODS ON A-VRP BENCHMARK PRoBlems

\begin{tabular}{|c|c|c|c|c|c|c|}
\hline SI. & Problem & $\begin{array}{l}\text { HHA } \\
{[13]}\end{array}$ & $\begin{array}{l}\text { Centroid-based } \\
\text { 3-phase [18] }\end{array}$ & $\begin{array}{l}\text { Sweep + Cluster } \\
\text { Adjust [18] }\end{array}$ & $\begin{array}{l}\text { Sweep Nearest } \\
{[19]}\end{array}$ & $\begin{array}{l}\text { Proposed Adaptive } \\
\text { Sweep + VTPSO }\end{array}$ \\
\hline 1 & A-n32-k5 & 1012 & 881 & 872 & 853 & 882 \\
\hline 2 & A-n33-k5 & 847 & 728 & 788 & 702 & 698 \\
\hline 3 & A-n33-k6 & 919 & 770 & 829 & 767 & 751 \\
\hline 4 & A-n34-k5 & 933 & 812 & 852 & 803 & 785 \\
\hline 5 & A-n36-k5 & 1126 & 814 & 884 & 840 & 881 \\
\hline 6 & A-n37-k5 & 876 & 756 & 734 & 797 & 754 \\
\hline 7 & A-n37-k6 & 1180 & 1027 & 1050 & 966 & 1112 \\
\hline 8 & A-n38-k5 & 920 & 819 & 874 & 801 & 813 \\
\hline 9 & A-n39-k5 & 1147 & 864 & 971 & 886 & 877 \\
\hline 10 & A-n39-k6 & 1065 & 881 & 966 & - & 972 \\
\hline 11 & A-n44-k6 & 1356 & 1037 & 1092 & 1020 & 1056 \\
\hline 12 & A-n45-k6 & 1210 & 1040 & 1043 & 991 & 1073 \\
\hline 13 & A-n45-k7 & 1361 & 1288 & 1281 & 1235 & 1305 \\
\hline 14 & A-n46-k7 & 1071 & 992 & 1013 & 1022 & 975 \\
\hline 15 & A-n48-k7 & 1292 & 1145 & 1143 & 1181 & 1152 \\
\hline 16 & A-n53-k7 & 1261 & 1117 & 1116 & - & 1090 \\
\hline 17 & A-n54-k7 & 1414 & 1209 & 1320 & - & 1361 \\
\hline 18 & A-n55-k9 & 1317 & 1155 & 1192 & 1134 & 1190 \\
\hline 19 & A-n60-k9 & 1733 & 1430 & 1574 & 1446 & 1503 \\
\hline 20 & A-n61-k9 & 1285 & 1201 & 1184 & 1158 & 1164 \\
\hline 21 & A-n62-k8 & 1604 & 1470 & 1559 & 1392 & 1408 \\
\hline 22 & A-n63-k9 & 2001 & 1766 & 1823 & 1763 & 1823 \\
\hline 23 & A-n63-k10 & 1542 & 1405 & 1523 & 1475 & 1477 \\
\hline 24 & A-n64-k9 & 1821 & 1587 & 1597 & 1586 & 1598 \\
\hline 25 & A-n65-k9 & 1429 & 1276 & 1351 & 1299 & 1317 \\
\hline 26 & A-n69-k9 & 1333 & 1283 & 1254 & 1225 & 1259 \\
\hline \multirow[t]{8}{*}{27} & A-n80-k10 & 2318 & 1883 & 2014 & 1896 & 2136 \\
\hline & Average & 1310.11 & 1134.67 & 1181.44 & 1134.92 & 1163.41 \\
\hline & Best/Worst & $0 / 27$ & $8 / 0$ & $2 / 0$ & $12 / 0$ & $5 / 0$ \\
\hline & & & \multicolumn{4}{|c|}{ Pairwise Win/Draw/Lose Summary } \\
\hline & HHA & - & $27 / 0 / 0$ & $27 / 0 / 0$ & $24 / 0 / 0$ & $27 / 0 / 0$ \\
\hline & Centroid-based 3-phase & & - & $7 / 0 / 20$ & $15 / 0 / 9$ & $10 / 0 / 17$ \\
\hline & Sweep + Cluster Adjust & & & - & $21 / 0 / 3$ & $15 / 1 / 11$ \\
\hline & Sweep Nearest & & & & - & $7 / 0 / 17$ \\
\hline
\end{tabular}

From Table III, it is observed that most of the cases adaptive Sweep outperformed its corresponding standard Sweep clustering. It is notable that for a particular problem, the outperformance of adaptive Sweep is only for different starting angle in Sweep because VTPSO is commonly used for vehicle route optimization in both the cases. As an example, for A-n33-k6 problem, standard Sweep (i.e, $\theta_{s}=0^{0}$ ) achieved CVRP cost of 874. For the same problem the outcome of adaptive Sweep with adaptively selected starting angle $303.18^{0}$ is 751 . Adaptive Sweep cluster outperformed standard Sweep cluster in 16 out of 27 cases. Standard Sweep is found better than adaptive Sweep for only A-n69-k9 problem. For the problem, standard Sweep achieved CVRP cost 1254 but adaptive Sweep achieved slightly larger CVRP cost which is
1259. On the basis of average CVRP cost over 27 problems, adaptive Sweep outperformed standard Sweep. The average CVRP costs for standard Sweep and adaptive Sweep are 1200.26 and 1163.41, respectively. In case of P-VRP benchmark problems, adaptive Sweep is also outperformed standard Sweep. The average CVRP costs for standard Sweep and adaptive Sweep are 645.38 and 637.17, respectively.

To identify the proficiency of proposed adaptive Sweep based approach, its outcomes have been compared with prominent CVRP methods. Among the selected methods, hybrid heuristic approach (HHA) [13], Sweep + Cluster Adjustment [18] and Sweep nearest [19] are also used Sweep based clustering to assign nodes to different vehicles but followed different approaches for route generation of 
individual vehicles. HHA [13] is the most recent CVRP method which used nearest neighbor method for route optimization. Centroid-based 3-phase [18] method is also considered in result comparison as it is found an effective method to solve similar benchmark CVRPs. The method follows three different steps: cluster formation with centroid based approach from the farthest point, centroid based cluster adjustment and finally route generation using Lin-Kernighan heuristic method.

Tables V and VI compare outcomes of adaptive Sweep based method with the selected existing methods in solving AVRP and P-VRP benchmark problems. The results for proposed adaptive Sweep + VTPSO are collected from Tables III and IV. On the other hand, presented results of the existing methods are the reported results in corresponding papers. If results are not available for problems with a particular existing method then those are marked as '-'. The best (i.e., minimum) CVRP cost among the five methods for a particular problem is marked as bold face type. Bottom of a table shows pairwise Win/Draw/Lose summary among the methods for better understanding. According to Table V, Centroid-based 3-phase is the overall best and HHA is the worst showing average CVRP cost of 1134.67 and 1310.11, respectively. On the other hand, proposed adaptive Sweep + VTPSO is shown competitive to Centroid-based 3-phase showing average CVRP cost 1163.41. The proposed method showed best CVRP solution for five cases and outperformed Centroid-based 3-phase for 10 cases out of 27 cases. More interestingly, the proposed method outperformed Sweep based HHA and Sweep + Cluster Adjust for 27 and 15, respectively.

TABLE VI. CVRP COST COMPARISON WITH EXISTING METHODS ON P-VRP BENCHMARK PROBLEMS

\begin{tabular}{|c|c|c|c|c|c|c|}
\hline Sl. & Problem & $\begin{array}{c}\text { HHA } \\
{[13]}\end{array}$ & $\begin{array}{c}\text { Centroid-based } \\
\text { 3-phase [18] }\end{array}$ & $\begin{array}{c}\text { Sweep + Cluster } \\
\text { Adjust [18] }\end{array}$ & $\begin{array}{c}\text { Sweep Nearest } \\
{[19]}\end{array}$ & $\begin{array}{l}\text { Proposed Adaptive } \\
\text { Sweep + VTPSO }\end{array}$ \\
\hline 1 & P-n16-k8 & 546 & 497 & 568 & 463 & 549 \\
\hline 2 & P-n19-k2 & 253 & 256 & 236 & 264 & 246 \\
\hline 3 & P-n20-k2 & 267 & 240 & 238 & 217 & 249 \\
\hline 4 & P-n21-k2 & 288 & 240 & 238 & 211 & 211 \\
\hline 5 & P-n22-k2 & 274 & 245 & 237 & 219 & 216 \\
\hline 6 & P-n22-k8 & 667 & 672 & 687 & 721 & 633 \\
\hline 7 & P-n23-k8 & 743 & 703 & 645 & 558 & 634 \\
\hline 8 & P-n40-k5 & 563 & 505 & 499 & 516 & 483 \\
\hline 9 & P-n45-k5 & 662 & 533 & 525 & - & 524 \\
\hline 10 & P-n50-k7 & 647 & 583 & 585 & - & 583 \\
\hline 11 & P-n50-k8 & 721 & 669 & 675 & - & 677 \\
\hline 12 & P-n50-k10 & 808 & 740 & 779 & - & 783 \\
\hline 13 & P-n51-k10 & 857 & 779 & 806 & - & 802 \\
\hline 14 & P-n55-k7 & 679 & 610 & 611 & - & 595 \\
\hline 15 & P-n55-k8 & 690 & 654 & 601 & - & 586 \\
\hline 16 & P-n55-k10 & 832 & 749 & 763 & - & 745 \\
\hline 17 & P-n55-k15 & 1180 & 1022 & 1056 & - & 1099 \\
\hline 18 & P-n60-k10 & 896 & 786 & 823 & - & 830 \\
\hline 19 & P-n60-k15 & 1159 & 1006 & 1086 & - & 1119 \\
\hline 20 & P-n65-k10 & 964 & 836 & 856 & - & 859 \\
\hline 21 & P-n70-k10 & 989 & 891 & 902 & - & 911 \\
\hline 22 & P-n76-k4 & 753 & 685 & 603 & 690 & 612 \\
\hline 23 & P-n76-k5 & 671 & 737 & 647 & - & 647 \\
\hline 24 & P-n101-k4 & 891 & 698 & 702 & 789 & 699 \\
\hline & Average & 708.33 & 639.00 & 640.33 & 464.8 & 637.17 \\
\hline & Best/Worst & $\mathbf{0} / \mathbf{2 0}$ & $10 / 1$ & $3 / 1$ & $4 / 2$ & 10/0 \\
\hline & & & \multicolumn{4}{|c|}{ Pairwise Win/Draw/Lose Summary } \\
\hline & HHA & - & $21 / 0 / 3$ & $22 / 0 / 2$ & $8 / 0 / 2$ & $23 / 0 / 1$ \\
\hline & Centroid-based 3-phase & & - & $10 / 0 / 14$ & $5 / 0 / 5$ & $12 / 1 / 11$ \\
\hline & Sweep + Cluster Adjust & & - & - & $5 / 0 / 5$ & $13 / 1 / 10$ \\
\hline & Sweep Nearest & & & & - & $6 / 1 / 3$ \\
\hline
\end{tabular}


The comparative results in Table VI identified the proposed adaptive Sweep + VTPSO as the best for P-VRP benchmark problems. The proposed method is shown the best for 10 cases out of 24 cases and achieved average cost of 637.17. The proposed method outperformed HHA, Centroidbased 3-phase, Sweep + Cluster Adjust, Sweep Nearest on 23, 12,13 and 6 cases, respectively, out of 24 cases. It is notable that Sweep Nearest tested only 10 problems. Between two existing Sweep based methods, HHA outperformed proposed method only for P-n16-k8 that is very small sized problem. Finally, outcomes of the proposed method revealed the proficiency of adaptive Sweep in clustering and VTPSO in route optimizing.

\section{CONCLUSIONS}

A two-phase CVRP solving method has been investigated through clustering with proposed adaptive Sweep and individual vehicle route optimizing with VTPSO. Adaptive Sweep is the extension of popular Sweep clustering where starting angle of cluster formation is determined through a heuristic approach based on nodes angle differences as well as distances from the depot. The experimental results on the benchmark problems revealed that adaptive Sweep is better than standard Sweep. Finally, proposed adaptive Sweep plus VTPSO is identified as a prominent CVRP solving method when outcomes compared with related existing methods in solving a large number of benchmark problems.

There are several future potential directions that follow from this study. In this study, angle difference and distance from the depot are considered to select starting angle. Scheme including node demand in selection criteria might improve performance and remain as future study. Moreover, it might be interesting to incorporate such motivation of cluster formation in other cluster first route second CVRP methods.

\section{REFERENCES}

[1] F. Daneshzand, "The Vehicle-Routing Problem," Logistics Operations and Management: Concepts and Models, Elsevier Insights, 2011.

[2] T. Carić, A. Galić, J. Fosin, et al. "A Modelling and Optimization Framework for Real-World Vehicle Routing Problems," Vehicle Routing Problem, In-The, 2008.

[3] G. Vaira, "Genetic Algorithm for Vehicle Routing Problem," PhD. Thesis, Vilnius University, Italy, 2014.

[4] C. Pornsing, "A Particle Swarm Optimization for the Vehicle Routing Problem," PhD. Thesis, University of Rhode Island, USA, 2014.

[5] N. M. Darani, V. Ahmadi, Z. S. Eskandari, M. Yousefikhoshbakht, "Solving the Capacitated Clustering Problem by a Combined Meta-
Heuristic Algorithm," Journal of Advances in Computer Research, vol. 4, no. 1, pp. 89-100, 2013.

[6] P. Boonsam and N. Suthikarnnarunai, "Assignment Problem and Vehicle Routing Problem for an Improvement of Cash Distribution," Proceedings of the World Congress on Engineering and Computer Science 2011, Vol II, WCECS 2011, October 19-21, 2011, San Francisco, USA.

[7] N. Suthikarnnarunai, "A Sweep Algorithm for the Mix Fleet Vehicle Routing Problem," Proceedings of the International MultiConference of Engineers and Computer Scientists 2008 Vol II, IMECS 2008, 19-21 March, 2008, Hong Kong.

[8] G. W. Nurcahyo, R. A. Alias, S. M. Shamsuddin and M. N. Md. Sap, "Sweep Algorithm in vehicle routing problem for public Transport," Journal Antarabangsa (Teknologi Maklumat), vol. 2, pp. 51-64, 2002.

[9] S. Han and Y. Tabata, "A Hybrid Genetic Algorithm for the Vehicle Routing Problem with Controlling Lethal Gene," Asia Pacific Management Review, vol. 7, no. 3, pp. 405-426, 2002.

[10] B. E. Gillett and L. R. Miller, "A heuristic algorithm for the vehicle dispatch problem," Operations Research, vol. 22, no. 2 pp. 340-349, 1974.

[11] M. Yousefikhoshbakht and E. Khorram, "Solving the vehicle routing problem by a hybrid meta-heuristic algorithm," Journal of Industrial Engineering International, vol. 8(11), 2012.

[12] M. Reed, A. Yiannakou and R. Evering, "An ant colony algorithm for the multi-compartment vehicle routing problem," Applied Soft Computing, vol. 15, pp. 169-176, 2014.

[13] M.. M. Aziz, H. A. El-Ghareeb and M. S. M. Ksasy, "Hybrid Heuristic Algorithm for solving Capacitated Vehicle Routing problem" International Journal of Computer and Technology, vol. 12, no. 9, pp. 3846-3851, 2014.

[14] S. R. Venkatesan, D. Logendran, and D. Chandramohan, "Optimization of Capacitated Vehicle Routing Problem using PSO," International Journal of Engineering Science and Technology (IJEST), vol. 3, pp. 7469-7477, 2011.

[15] M. A. H. Akhand, S. Akter, M. A. Rashid and S. B. Yaakob, "Velocity Tentative PSO: An Optimal Velocity Implementation based Particle Swarm Optimization to Solve Traveling Salesman Problem," IAENG International Journal of Computer Science, vol. 42, no.3, pp 221-232, 2015.

[16] Augerat et al., "Capacitated Vehicle Routing Problem Instances," Networking and Emerging Optimization (NEO) Research Group, University of Malaga, Spain.

[17] M. A. H. Akhand, Zahrul Jannat, Tanzima Sultana and Al-Mahmud, "Optimization of Capacitated Vehicle Routing Problem using ProducerScrounger Method," in Proc. of 2015 IEEE International WIE Conference on Electrical and Computer Engineering (WIECON-ECE 2015), BUET, Dhaka, Bangladesh, pp. 297-300, December 19-20, 2015.

[18] K. Shin and S. Han, "A Centroid-Based Heuristic Algorithm for the Capacitated Vehicle Routing Problem," Computing and Informatics, vol. 30, pp. 721-732, 2011.

[19] B. Na, Y. Jun and B. Kim, "Some Extensions to the Sweep Algorithm," Int J Adv Manuf Technol, vol. 56, pp. 1057-1067, 2011. 